\title{
ANTI-MICROBIAL EFFECT OF COMBINATIONS OF COLISTIN METHANESULFONATE AND CHLORAMPHENICOL. I IN VITRO EFFEGT
}

\author{
Masaya Kawakami, Yutaka Nagai*, Shoji Shimizu* \\ and Susumu Mitsuhashi \\ Department of Microbiology, School of Medicine, Gunma University, \\ Maebashi, Japan
}

(Received for publication June 9, 1971)

\begin{abstract}
Antimicrobial activity in vitro of colistin methanesulfonate and chloramphenicol was studied, using gram-negative enterobacteria and Staphylococcus aureus with various drug-resistance patterns and serotypes or phage types. A synergistic growth inhibitory action of both drugs against most of the gram-negative bacteria was demonstrated by a qualitative method and confirmed by a quantitative determination and statistical evaluation. It was also found that the combined action of both drugs obviously suppressed the development of drug resistant mutants of gram-positives as well as of gram-negative microorganisms.
\end{abstract}

Colistin and chloramphenicol are well known antibiotics acting on gram-negative bacteria, especially Enterobacteria. Both antibiotics completely differ in their chemical and biological properties. Chloramphenicol (CP) has a low toxicity and a wide antimicrobial spectrum; on the other hand, colistin methanesulfonate (CLM) seldom gives rise to the appearance of resistant mutants ${ }^{1}$. It is interesting to know whether one can off set the shortcomings of one antibiotic against the merits of the other by a combination of both drugs. This paper concerns with an attempt to make an improved drug by the combination of CLM and CP.

\section{Materials and Methods}

Test microorganisms: One hundred strains out of a total of about 6,000 strains of staphylococci, isolated from lesions in man, and collected, stored, and maintained by the Research Committee of Drug-Resistance in Staphylococci (Chief: Dr. Tokuji Ichikawa) were studied. The strains selected have different phage types and resistance patterns to antibiotics including kanamycin, chloramphenicol, macrolide group antibiotics, synthetic penicillins and cephalosporins.

Six strains of dysentery bacilli of different serotypes and resistance to various antibiotics (including single and multiple resistance to $\mathrm{CP}$, tetracycline, streptomycin, kanamycin and sulfonamides) were selected from 20,000 strains which had been isolated by the Research Committe for Drug-Resistant Shigella Strains (Chief : Dr. Tadato Ezaki), and stored in our laboratory.

Gram-negative rods other than dysentery bacilli were representative multiple antibioticresistant strains maintained in our laboratory.

Qualitative demonstration of synergism by the plate technique : The usual agar dilu-

* Present address : Kayaku Antibiotics Research, Co., Ltd., Tokyo 
tion method $^{2}$ was used for the determination of the maximum drug concentration still allowing bacterial growth (MAC).

A slight modification of the crossed paper strip method reported by $\mathrm{CHABBERT}^{3)}$ was used for the demonstration of synergism. Two-tenth $\mathrm{ml}$ of an 18-hour culture of test bacteria was mixed with $5 \mathrm{ml}$ of soft agar in BHI broth and poured on HI agar. Four strips of filter paper (No. 6, Toyo Filter Papar Co., Tokyo), each $5 \times 45 \mathrm{~mm}$, were dipped in drug solutions and placed on the agar plate, forming a square with sides of $45 \mathrm{~mm}$ (Plate 1). The plates were placed for 4 hours at $4^{\circ} \mathrm{C}$ and then for 18 hours at $37^{\circ} \mathrm{C}$. Two strips containing the same kind but different concentrations of a drug were placed in one corner of the square, and the other two containing another drug were arranged at the opposite corner. Curves of the inhibitory zone at the corner of paper strips containing heterologous drugs were compared with those containing homologous drugs. When a smaller curvature in the heterologous corners was observed in comparison with the mean of homologous controls, the heterologous drugs were considered to have a synergistic action ${ }^{4}$.

Assay of the appearance of drug resistant mutant: About $10^{9}$ bacteria were inoculated on $\mathrm{HI}$ agar plates containing a drug concentration higher than the MAC value. Colonies, less than 10 in number, which had developed after 48 -hour incubation at $37^{\circ} \mathrm{C}$, were regarded as resistant mutants.

Quantitative determination of synergism: One $\mathrm{ml}$ of an overnight culture of bacteria in $\mathrm{BHI}$ broth was transferred to $10 \mathrm{ml}$ of fresh broth and incubated with shaking at $37^{\circ} \mathrm{C}$ until an optical density (O.D.) at $450 \mathrm{~m} \mu$ of 0.8 was reached. One-tenth $\mathrm{ml}$ of this culture was then added to $10 \mathrm{ml}$ of fresh broth containing various amounts of drugs at certain combination ratios. The incubated cultures were placed in a tube suitable for photometric turbidity measurement and then incubated at $37^{\circ} \mathrm{C}$. The optical density at $450 \mathrm{~m} \mu$ was measured and recorded continuously by using an automatic recording photometer (Biophotometer, Jasco-Jouan, Paris, France). From the bacterial concentration, expressed as O.D. obtained after 120 minutes of incubation, the growth rate (GR) was calculated and expressed as percentage of that obtained in the drug-free control tube:

$$
\mathrm{GR}=\frac{E_{d 2}-E_{d 0}}{E_{c 2}-E_{c 0}} \times 100
$$

where $E_{d 2}$ and $E_{d 0}$ were O.D. in the drug-containing tube at 2 hours and 0 hour, $E_{c 2}$ and $E_{c 0}$ were O.D. in the control tube at 2 hours and 0 hour, respectively.

Statistical methods: Growth rates at different drug concentrations were plotted against the doses in a log scale diagram (Fig. 3). A reciprocal relationship between the growth rates and doses was observed. From the regression line obtained by the least square method, the $70 \%$ effective dose $\left(\mathrm{ED}_{70}\right)$ and its $95 \%$ confidence limits were calculated by the method of WAITZ and DRESNER ${ }^{5}$.

When a combined drug produces an additive effect, its potency $(P a)$ is expressed as the sum of potencies $\left(P_{1}+P_{2}\right)$ of the single drugs.

The potency of a drug is expressed as a reciprocal of the effective dose :

$$
\frac{1}{D a}=\frac{R_{1}}{D_{1}}+\frac{R_{2}}{D_{2}}
$$

where $D a$ is the effective dose of combined drug which reveals additive effect, $D_{1}$ and $D_{2}$ are experimentally determined effective doses, and $R_{1}$ and $R_{2}$ are composition rate of each of drugs in culture medium*.

To determine whether the combined drug reveals an additive or synergistic effect, we calculated a ratio (SR) of exprimentally determined potency $(P d)$ to a hypothetical potency in which additive effect was assumed $(\mathrm{Pa})^{* *}$ :

\footnotetext{
* $R_{1}$ and $R_{2}$ are expressed as $\frac{C_{1}}{C_{1}+C_{2}}$ and $\frac{C_{2}}{C_{1}+C_{2}}$ respectively, where $C_{1}$ and $C_{2}$ are the concentrations of the single drugs which compose the effective dose of the combined drug.

** The SR corresponds to the reciprocal of FIC index ${ }^{6}$.
} 


$$
\mathrm{SR}=\frac{P d}{P a}=\frac{D a}{D d}
$$

When the SR is 1 , the combined drug is thought to have an additive effect, and when the $\mathrm{SR}$ is more than 1 , the drug is decided to have a synergistic effect. This ratio, indicating the degree of the synergistic activity of a combined drug, was designated as synergistic ratio.

Inserting the equation (2) to (3),

$$
\mathrm{SR}=\frac{1}{\left(\frac{R_{1}}{D_{1}}+\frac{R_{2}}{D_{2}}\right) D d}
$$

When the exponents of doses are used to obtain linearly correlated results in a doseresponse diagram, confidence limits of doses are expressed as $D / f_{D}$ and $D \cdot f_{D}$, where the error function, $f_{D}=\operatorname{antilog} 1.96 \sigma_{\log D}\left(\sigma_{\log D} \text { is standard error of } \log D\right)^{7}$.

The error function for the determination of the $95 \%$ confidence limits of $D a$ is calculated by using the following equation:

$$
f_{D a}=1+\sqrt{\frac{\left[\frac{R_{1}}{D_{1}}\left(f_{D_{1}}-1\right)\right]^{2}+\left[\frac{R_{2}}{D_{2}}\left(f_{D_{2}}-1\right)\right]^{2}}{\left(\frac{R_{1}}{D_{1}}\right)^{2}+\left(\frac{R_{2}}{D_{2}}\right)^{2}}}
$$

The confidence limits are calculated as $D a / f_{D a}$ and $D a \cdot f_{D a}$. The equation (5) is derived as follows :

Generally, standard error of $A+B$ is :

$$
\sigma_{A+B}=\sqrt{\sigma_{A}^{2}+\sigma_{B}^{2}}
$$

The $f_{D}$ must be transferred to the form of $\sigma_{D}$ for the calculation of standard error of addition such expressed as equation (6). When the $f_{D}$ is less than 1.5,

$$
\begin{aligned}
& f_{D} \doteqdot 1+1.96 \sigma_{D} / D \\
& 1.96 \sigma_{D} \fallingdotseq D\left(f_{D}-1\right)
\end{aligned}
$$

The standard error of $K / D$ ( $K$ is a constant) is similarly expressed as :

$$
1.96 \sigma_{K / D} \doteqdot \frac{K}{D}\left(f_{D}-1\right)
$$

Thus,

$$
1.96 \sigma_{\frac{R_{1}}{D_{1}}+\frac{R_{2}}{D_{2}}} \doteqdot \sqrt{\left[\frac{R_{1}}{D_{1}}\left(f_{D_{1}}-1\right)\right]^{2}+\left[\frac{R_{2}}{D_{2}}\left(f_{D_{2}}-1\right)\right]^{2}}
$$

The $f_{D a}$ is derived from (6) and (7):

When the amounts of single drugs $\left(C_{1}\right.$ and $\left.C_{2}\right)$, which compose the effective dose of the combined drug $(D d)$, are konwn, a more simple equation can be used for the SR:

because

$$
\mathrm{SR}=\frac{1}{\frac{C_{1}}{D_{1}}+\frac{C_{2}}{D_{2}}}
$$

In this case,

$$
\begin{gathered}
D d=C_{1}+C_{2} \\
R_{1}=\frac{C_{1}}{C_{1}+C_{2}} \text { and } R_{2}=\frac{C_{2}}{C_{1}+C_{2}}
\end{gathered}
$$

$$
\begin{gathered}
D a=\frac{C_{1}+C_{2}}{\frac{C_{1}}{D_{1}}+\frac{C_{2}}{D_{2}}} \\
f_{D a}=1+\sqrt{\frac{\left[\frac{C_{1}}{D_{1}}\left(f_{D_{1}}-1\right)\right]^{2}+\left[\frac{C_{2}}{D_{2}}\left(f_{D_{2}}-1\right)\right]^{2}}{\left(\frac{C_{1}}{D_{1}}\right)^{2}+\left(\frac{C_{2}}{D_{2}}\right)^{2}}}
\end{gathered}
$$




$$
\begin{aligned}
f_{D a} & =f_{1 / D a}=f_{\substack{R_{1-1}-R_{2} \\
D_{1}+D_{2}}} \\
& \doteqdot 1+\frac{\sqrt{\left[\frac{R_{1}}{D_{1}}\left(f_{D_{1}}-1\right)\right]^{2}+\left[\frac{R_{2}}{D_{2}}\left(f_{D_{2}}-1\right)\right]^{2}}}{\frac{R_{1}}{D_{1}}+\frac{R_{2}}{D_{2}}}
\end{aligned}
$$

However, $f_{D a}$ value expressed by the equation (8) seems to be too small in some cases of combination of $R_{1}, R_{2}, D_{1}$ and $D_{2}$ because $D\left(f_{D}-1\right)$ is somewhat smaller than actual value of $1.96 \sigma_{D}$ (e.g. when $f_{D 1}=f_{D 2}=1.2$ is put into the equation (8), $f_{D a}<1.2$ is obtained in the case $R_{1}<R_{2}, D_{1}<D_{2}$ ). To eliminate this effect, the denominator of equation (8) was modified. The equation (5), thus obtained, was used for the calculation of $f_{D a}$.

The following equation, cited by Litchfield and $\mathrm{W}_{\text {ILCOXON }}{ }^{7)}$, was used for the calculation of $f_{\mathrm{SR}}$ :

$$
f_{\mathrm{SR}}=\operatorname{antilog} \sqrt{\left(\log f_{D a}\right)^{2}+\left(\log f_{D d}\right)^{2}}
$$

and the $95 \%$ confidence limits of synergistic ratio were calculated as $\mathrm{SR} / f_{\mathrm{SR}}$ and $\mathrm{SR} \cdot f_{\mathrm{SR}}$.

\section{Results}

Effect of Combined Drugs Determined by Plate Technique

Various strains of gram-negative bacteria were inoculated on BHI agar plates containing CLM and CP in combinations and in varying amounts. Depending on the sensitivity of the strains, various patterns of growth inhibition were observed as shown in Fig. 1 and Table 1. When the size of inoculum was $10^{4}$ per plate, obvious synergism of both drugs was found with a few strains such as Shigella flexneri 3a. However, obvious synergism could not be demonstrated with other strains by this plate method. The inhibitory effect of a combination of both drugs on the development of resistant mutants was easily demonstrated by this plate method. Some examples of the results are given in Table 2 .

Fig. 1. Inhibition of growth of drug sensitive bacteria on agar plates by various combination ratios of CLM and CP.

+ : positive growth using an inoculum of $10^{6}$ bacteria. $\oplus$ : positive growth using an inoculum of $10^{4}$ bacteria. Drug concentrations are plotted on the ordinate (CLM) and abscissa (CP).
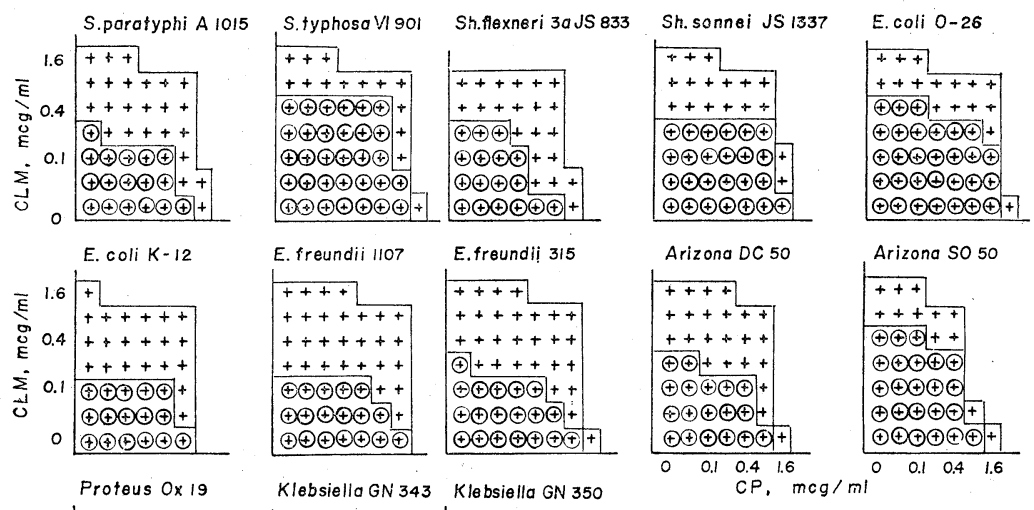

E.froundii 315
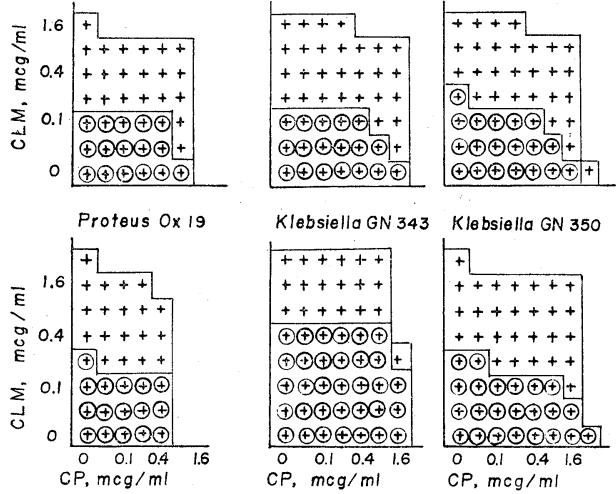

$++++++$

$++++++$

$\oplus++++++$

$\oplus \oplus \oplus \oplus \oplus \oplus$

$\oplus \oplus \oplus \oplus \oplus \oplus \oplus^{+}+$
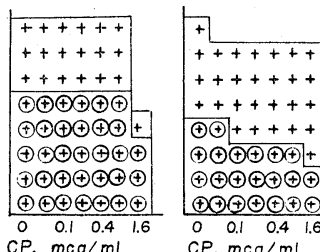

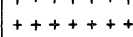

$++++++$

$\oplus \oplus \oplus \oplus \oplus(\oplus)+$

$\oplus \oplus \oplus \oplus \oplus \oplus \oplus)$
$\oplus \oplus \oplus \oplus \oplus \oplus \oplus \oplus)$

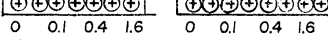

$\mathrm{CP}, \mathrm{meg} / \mathrm{ml}$ 
Table 1. Inhibition of growth of CLM or CP resistant strains by combined drugs

\begin{tabular}{l|c|c|c|c|c}
\hline \multirow{2}{*}{ Strains $*$} & \multicolumn{6}{|c}{ Ratios of CLM to CP in plates } \\
\cline { 2 - 6 } & $1: 0$ & $2: 1$ & $1: 1$ & $1: 2$ & $0: 1$ \\
\hline Shigella flexneri 2a $\mathrm{R}_{4}$ (JS2997) & 0.4 & 0.4 & 0.4 & 1.6 & $>100$ \\
S. flexneri 3a $\mathrm{R}_{4}$ (JS2529) & 0.1 & 0.1 & 0.1 & 0.2 & $>100$ \\
S. sonnei $\mathrm{R}_{4}$ (JS949) & 0.8 & 0.1 & 0.1 & 0.2 & $>100$ \\
Proteus (OX2) & $>100$ & 0.4 & 0.2 & 0.2 & 0.2 \\
Pseudomonas pyogenes (GN83) & 3.2 & 3.2 & 3.2 & 6.3 & $>100$ \\
\hline
\end{tabular}

Each number indicates the MAC of the combined drugs expressed in mcg per ml medium.

$*: \mathrm{R}_{4}$ means multiple resistance to four antibiotics due to presence of $\mathrm{R}$ factor. (TC, CP, SM, SA)

Table 2. Effect of combined drugs on the appearance of drug resistant mutants using the agar plate technique

Staphylococcus MS-353

\begin{tabular}{c|c|c|c|c|c|c}
\hline \multirow{2}{*}{$\begin{array}{c}\mathrm{CP} \\
(\mathrm{mcg} / \mathrm{ml})\end{array}$} & \multicolumn{7}{c}{$\mathrm{CLM}$} & $(\mathrm{mcg} / \mathrm{ml})$ \\
\cline { 2 - 7 } & 25 & 12.5 & 6.25 & 3.2 & 1.6 & 0 \\
\hline 25 & - & - & - & - & 1 & $2 *$ \\
12.5 & - & - & - & - & 2 & $1 *$ \\
6.25 & - & - & - & - & + & + \\
3.2 & - & - & + & + & + & + \\
1.6 & - & + & + & + & + & + \\
0 & + & + & + & + & + & + \\
\hline
\end{tabular}

E. coli $\mathrm{K}-12$

\begin{tabular}{c|c|c|c|c|c|c|c|c|c|c|c}
\hline \multirow{2}{*}{$\mathrm{CP} \quad(\mathrm{mcg} / \mathrm{ml})$} & \multicolumn{10}{c}{ CLM } & \multicolumn{1}{c}{ (mcg/ml) } \\
\cline { 2 - 8 } & 50 & 25 & 12.5 & 6.25 & 3.2 & 1.6 & 0.8 & 0.4 & 0 \\
\hline 50 & - & - & - & - & - & - & - & - & - \\
25 & - & - & - & - & - & - & - & - & - \\
12.5 & - & - & - & - & - & - & - & - & $2 *$ \\
6.25 & - & - & - & - & - & - & - & - & $1 *$ \\
3.2 & - & - & - & - & - & - & - & - & - \\
1.6 & - & - & - & - & - & - & - & - & + \\
0.8 & - & - & - & - & - & - & - & + & + \\
0.4 & - & - & - & + & + & + & + & + & + \\
0
\end{tabular}

Inoculum size : $10^{9}$ bacteria per plate.

+ : Massive growth of bacteria after an overnight incubation.

*: Number indicates number of colonies observed on the plate.

Table 3. Qualitative determination of synergistic inhibition with CLM and CP using the strip method

\begin{tabular}{|c|c|c|c|c|c|c|}
\hline Strain & Synergism & Strain & Synergism & \multicolumn{2}{|c|}{ Strain } & Synergism \\
\hline S. paratyphi A 1005 & + & E. coli $0-111$ & + & Staphylococcus & MS-353 & ? \\
\hline S. paratyphi B 8006 & + & E. coli $\mathrm{K}-12$ W3630 & + & Staphylococcus & S-528 & ? \\
\hline S. typhosa V1901 & + & E. freundii Bal-107 & + & "1 & S-1107 & - \\
\hline Sh. flexneri 2a JS700 & + & Arizona SO-50 & + & "1 & S-774 & ? \\
\hline Sh. flexneri 3a JS2529 & + & Arizana D.C. 5 & + & $\prime \prime$ & S-784 & - \\
\hline Sh. flexneri 3a JS833 & + & Proteus OX19 & - & "1 & S-251 & - \\
\hline Sh. sonnei JS949 & + & Proteus $\mathrm{OX} 2$ & ? & "l & S-929 & + \\
\hline E. coli $0-26$ & + & Klebsiella GN343 & + & "I & S-1115 & ? \\
\hline
\end{tabular}

?: The inhibition zone was not clear, the mode of action could not be determined with certainty. 
Synergistic growth inhibitory effects of combined drugs could be demonstrated far better by the crossed paper strip method. Synergism was observed with 15 out of 17 gramnegative strains, including drug resistant ones as shown in Table 3 and Plate 1 . In the case of Proteus OX2, the inhibitory zone was not sharp, and it was difficult to determine the mode of drug action. A typical synergistic pattern of inhibition developed only in one out of 8 Staphylococcus strains tested.

Quantitative

Determination of

Synergism

Varying concentrations of drugs added to the culture medium of E. coli resulted in growth curves, shown in Fig. 2.

There was a reciprocal relationship between the growth rate and of the drug concentration near the $\mathrm{ED}_{70}$, as shown in Fig. 3, and the regression lines were shown tofit in well with the plotted points

Fig. 2. Growth curves of E. coli GN2411 in BHI broth containing various combinations of CLM and CP.

About $10^{5}$ bacteria in $10 \mathrm{ml}$ of $\mathrm{BHI}$ broth were incubated at $37^{\circ} \mathrm{C}$. The optical density was measured and recorded automatically by the Biophotometer. Number on each curve represents the concentration of drugs in $\mathrm{mcg} / \mathrm{m} 1$.

CLM

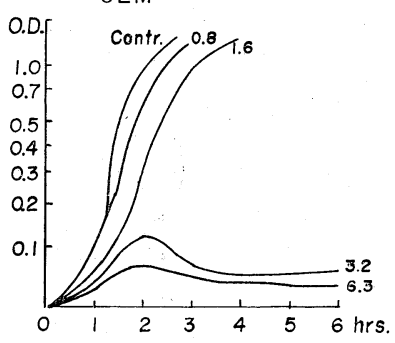

Plate 1. Synergistic action of CLM and CP. The number on each paper strip represents the concentration of drugs in $\mathrm{mg} / \mathrm{ml}$.

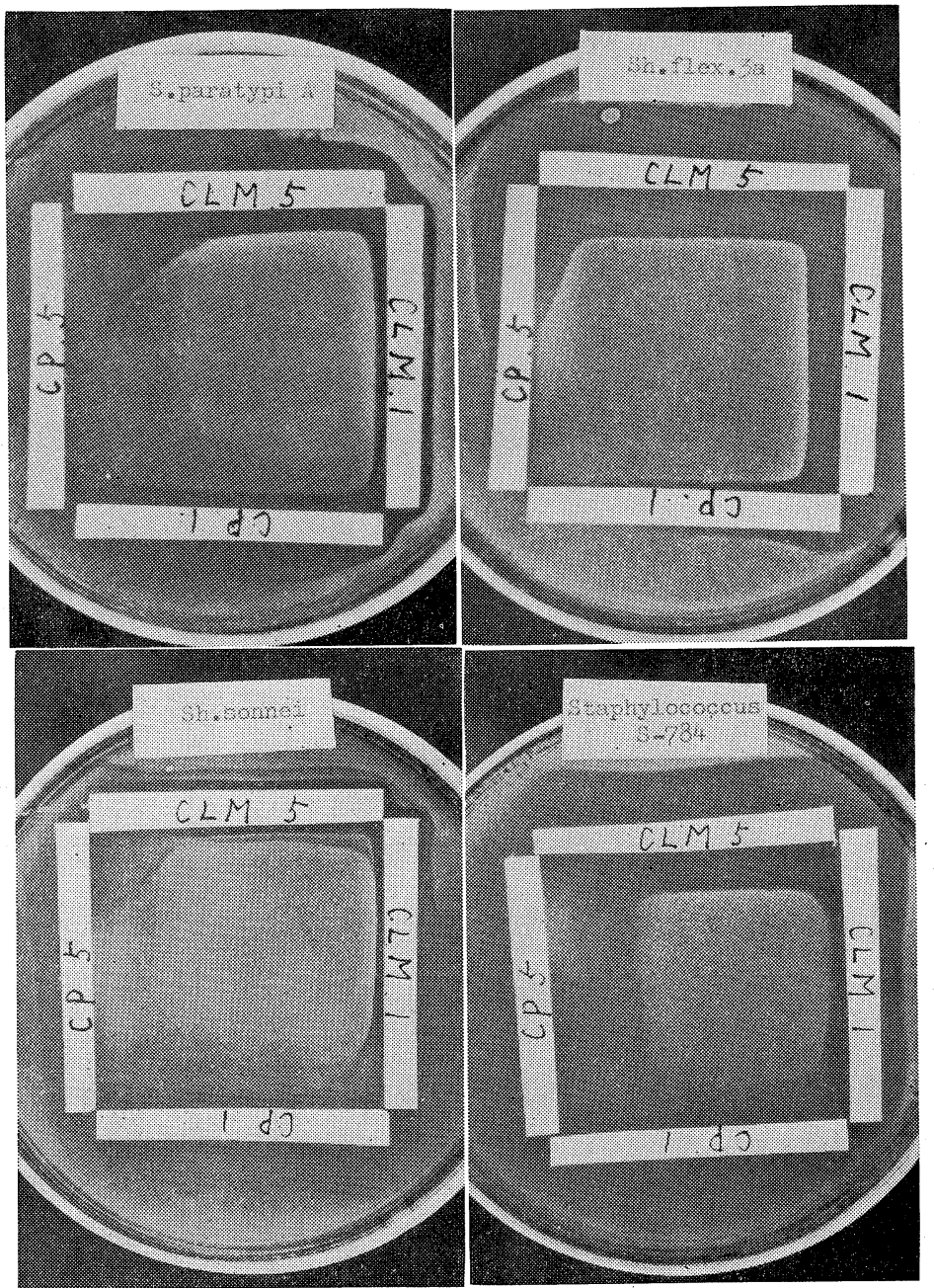


of each group by a $\chi^{2}$ test $(P=0.05)$. $\mathrm{ED}_{70}$ and its confidence limits calculated from this line are summarized in Table 4.

The $\mathrm{ED}_{70}$ instead of $\mathrm{ED}_{50}$ was determined for the evaluation of therapeutic effect because photometric measurements are more accurate at lower concentrations of bacteria (see Fig. 2), and the plotted points of the determined values scattered centering around the $\mathrm{ED}_{70}$ as shown in Fig. 3.

Table 4. Effective dose of single and combined drugs (CLM-CP, $2: 10$ )

\begin{tabular}{l|l|c|c|c|c}
\hline \multirow{2}{*}{ Groups } & \multicolumn{2}{c|}{ Regression constants $(\mathrm{log})$} & \multirow{2}{*}{$\begin{array}{c}\mathrm{ED}_{70} \\
(\mathrm{mg} / \mathrm{ml})\end{array}$} & 95\% confidence limits \\
\cline { 2 - 5 } & $\mathrm{a}$ & $\mathrm{b}$ & 1.82 & $1.41 \sim 2.36$ \\
\multirow{3}{*}{ Determined* } & $\mathrm{CLM}$ & -0.39 & 1.25 & 0.67 & $0.60 \sim 0.75$ \\
& $\mathrm{CP}$ & -0.73 & 0.06 & 0.30 & $0.24 \sim 0.38$ \\
\hline Hypothetical** & $\mathrm{CLM}+\mathrm{CP}$ & -1.05 & 1.01 & 0.74 & $0.63 \sim 0.89$ \\
\hline
\end{tabular}

* Experimentally determined values. **. A hypothetical value assuming an additional effect of the combined drugs (see text).

The results given in Table 4 show that the experimentally determined $\mathrm{ED}_{70}$ of a combined drug is smaller than the hypothetical $\mathrm{ED}_{70}$ in assuming an additive eff ect of combined drug. The synergistic ratio of combined drug was 2.5 , and its $95 \%$ confidence limits was 1.9 and $3.3\left(f_{\mathrm{SR}}=1.32\right)$. This means that the determined potency of the combined drug is approximately 2 to 3 times higher than the hypothetical potency in assuming an additive effect, and that a combination of CLM and CP produces a synergistic inhibitory effect on the growth of E. coli GN2411.

\section{Discussion}

Treatment with a combination of different drugs is useful when the combination have the following properties: (1) appearance of anti-microbial effect with microorganisms on which each of the single drug is ineffective; (2) lowered effective dose due to their synergistic action; and (3) prevention of the development of resistant mutants. Present results demonstrate that there is a synergistic growth inhibitory action of CLM and CP against most of the gram-negative enteric bacteria. This fact is in agreement with the observation of Mouton and Koelman ${ }^{4}$, and the present quantitative studies confirm this synergism of the two drugs. It was also found that the combination of both drugs suppresses the development of drug resistant mutants of gram-positive as well as of gramnegative species.

PatTe et al. ${ }^{8)}$ reported a method for the quantitative determination of drug synergism by which bacterial growth in liquid media containing various concentrations of drug was measured photometrically. Our simplified modification of their method and the statistical evaluation were described in the present paper. A suitable incubation period could be selected easily, and the bacterial concentration could be determined accurately for the com- 
parison of growth rates from the growth curves recorded by an automatic photometer.

A linear relationship between dose and growth rate plotted on a finit difference diagram has been demonstrated, and the logical fittness of these parameters in this diagram has also clearly been explained ${ }^{9)}$. However, this method is not suitable for practical use because of its complicacy of calculation procedures and difficulty in comparison of drug potencies. By a simple statistical examination, it was shown in the present paper that the growth rate plotted as function of the dose fitted well a straight line. Once this straight line relationship was evidenced, values for the determination of the accuracy of the effective dose can easily be obtained using the equations cited by WAITZ and DRESNER ${ }^{5}$. It is suggested that the present procedure may represent a useful method for the comparative study of antimicrobial agents, especially of combined drugs.

\section{Acknowledgement}

We wish to thank Prof. T. Tsusr, Department of Public Health, Gunma University, for his helpful advice on statistical studies and kind permission to use the computer.

\section{References}

1) Sous, H.; H. Löschner, F. LAGLer \& H. MüCKTER: Experimentelle Untersuchungen über das Antibioticum, Colistin. Arzneim-Forsch. $11: 395 \sim 400,1961$

2) Harada, K.; M. Suzuki, M. Kameda \& S. Mitsuinashi : Transmission of drug-resistance among Enterobacteriaceae. Japan. J. Exp. Med. $30: 289 \sim 299,1960$.

3) Chabbert, Y.: Une technique nouvelle d'étude de l'action bactériocide des associations d' antibiotiques : le transfert sur cellophane. Ann. Inst. Pasteur $93: 289 \sim 299,1957$

4) Mouton, R. P. \& A. Koelman : Bacteriostatic and bactericidal action of combined antibacterial agents in vitro. Antimicr. Agents \& Chemoth.-1965:261 266, 1966

5) W.aitz, J. A. \& A. J. Dresner : Selected statistical methods in chemotherapy. Adv. Chemotherapy $3: 1 \sim 37,1968$

6) Bushiy, S. R. M. \& G. H. Hrtchings : Trimethoprim, a sulphonamide potentiator. Brit. J. Pharmacol. Chemotherapy $33: 72 \sim 90,1968$

7) Litchfield, J. T. \& F. Wilcoxon: A simplified method of evaluating dose-effect experiments. J. Pharmacol. \& Exp. Therap. $96:$ 99 113, 1949.

8) Patte, J. C.; H. Hirsch \& Y. Chabbert : Étude des courbes d' effet bactériostatique des associations d' antibiotiques. Ann. Inst. Pasteur $94: 621 \sim 635,1958$

9) SASAKI, S. : On the growth curve of bacteria and a rapid assay method of penicillin. J. Antibiotics $4: 155 \sim 162,1950$ 